\title{
Enhancing the Authentication Scheme to Auditing the Cloud Storage and Security
}

\author{
K.Deepa ${ }^{a}$, S.Hariharan ${ }^{b}$, K.Kannan ${ }^{c}$ and R.Kavin ${ }^{d}$ \\ a \\ Department of Computer Science and Engineering, M.Kumarasamy College of \\ Engineering, Karur, Tamil Nadu, India - 639113 \\ b,c,d Department of Computer Science and Engineering, M.Kumarasamy College of Engineering, Karur, Tamil Nadu, India - \\ 639113
}

Article History: Received: 11 January 2021; Accepted: 27 February 2021; Published online: 5 April 2021

\begin{abstract}
Cloud computing has become a reality with new IT infrastructure based on several techniques such as distributed computing, virtualization, etc. Besides the many benefits that they can offer, cloud computing also comes with the difficulty of protecting data security. This paper first explores the basic concepts and analyzes the main aspects of data security about cloud computing. We then look at each problem, discussing its nature and existing solutions, if any. In particular, we will pay special attention to protecting data confidentiality/integrity/availability, data access, and monitoring, and complying with rules and obligations to ensure data security and confidentiality. With the fast advancement of organizing and portable gadgets, we are confronting a dangerous incensement of swarm sourced information. Existing frameworks as a rule depend on a confided in server to total the spatio fleeting publicly supported information and after that apply differential security component to bother the total insights previously distributing to give solid protection ensure. We propose a Modified appropriated specialist based protection saving structure, called MDADP that presents another dimension of various operators between the clients and the untrusted server.
\end{abstract}

Keywords: deduplication, Cloud capacity inspecting, deduplication, solid protection assurance, information security, cloud capacity

\section{Introduction}

Cloud computing is a modern IT infrastructure that makes computer resources accessible as a service to cloud users. Cloud computing provides scalable, on-demand, and measured services to cloud users anywhere, wherever, wherever the Internet is open and enables them to enjoy the imaginary limitless computing power by combining techniques such as Service Oriented Architecture (SOA), virtualization, disbursed computing, and others[1]. The cloud's services can be found at various levels of the device stack. This is referred to as X as a Service (XaaS), where $\mathrm{X}$ can refer to software, infrastructure, hardware, platforms, and so on.

For instance, Amazon EC2 provides Infrastructure as a Service, allowing cloud customers to manage nearly the entire software stack above the OS kernel; Google App Engine provides Software as a Service for conventional web applications, and Microsoft Azure provides services that are intermediate between App Engine and EC2.

Cloud customers can have huge and resilient IT resources without having to invest large amounts of money to develop their own data centers by handing over packages inside the cloud [2]. This reality would greatly benefit the IT industry, especially small and medium IT businesses, as well as individuals who have been severely limited by computing resources. As a result, cloud computing is expected to influence the IT industry in the future.

All these current frameworks center around utilizing cryptography or differential security to scramble or bother crude information on the information patron, which can ensure the genuine information independently, however isn't reasonable for the insurance of total measurements over publicly supported information, since the annoyance of crude information on every client would not influence the measurement estimation over publicly supported information. What's more, all current calculations under an untrusted server can't give solid assurance to constant information distributing. These issues rouse us to structure another differentially private system for continuous publicly supported measurable information distributing with the untrusted server.

\section{Related work}

Cloud computing to realize a total definition of what a Cloud is, utilizing the most characteristics ordinarily related with this worldview within the writing. More than 20 definitions have been considered permitting for the extraction of an agreement definition as well as the least definition containing the fundamental characteristics. This paper pays much consideration to the Framework worldview because it is frequently confounded with Cloud advances. We moreover de- copyist the relationships and qualifications between the Framework and Cloud approach. Clouds don't have a clear and total definition within the writing, however, which is a vital errand that will offer assistance to decide the zones of investigation and investigate new application spaces for the utilization of the Clouds. To handle this issue, the most accessible definitions extricated from the writing have been analyzed to supply both an integrator and an fundamental Cloud definition.

We propose an unused decentralized get-to-control conspire for secure information capacity in clouds that underpins mysterious verification. Within the proposed plot, the cloud confirms the realness of the arrangement without knowing the user's personality sometimes recently putting away information. We conspire too has the 
included include of getting to control in which as it were substantial clients are able to decode the put-away data. The conspire anticipates replay assaults and underpins creation, alteration, and reading information put away within the cloud. We to address client denial. Additionally, our verification and get to control conspire is decentralized and vigorous, not at all like other get to control plans outlined for clouds that are centralized. The communication, computation, and capacity overheads are comparable to centralized approaches.

Information sharing has never been simpler with the progress of cloud computing, and an exact examination of the shared information gives a cluster of benefits to both society and people. Information sharing with a huge number of members must take under consideration a few issues, counting efficiency, information judgment, and protection of data owner. Ring signature may be a promising candidate to build a mysterious and true informationsharing framework. It permits an information proprietor to namelessly verify his information which can be put into the cloud for capacity or examination reason.

\section{Literature survey}

Deduplication could be a famous technique in cloud capacity, wherein the most excellent generation of the repetitive records is spared with inside the cloud, irrespective of what number of customers need to download that file. Data Deduplication in Cloud Computing Cloud computing is a paradigm shift in Internet technologies [3][4]. Data deduplication can save storage space and reduce data transmission bandwidth. Public cloud garage auditing with deduplication is secure and consistent in price. Interior the cloud carport, a deduplication machine is utilized to abbreviate the carport length of the labels for judgment checks. Information Proprietorship Security by Outsourced Information Exchange With the quick headway of cloud computing, a developing number of businesses are picking to outsource their measurements and store them within the open cloud [5]. When parts of a company's commerce are obtained by another company, the relevant data is transferred to the acquiring company. In general, it is crucial to have verifiable ownership of the data by uploading the transfer data (DTPDP) to investigate how the processing costs of data transfer can be outsourced to the cloud, such as maintaining the quality of the data obtained remotely.

For the first time, we present a new definition in this paper, DT-PDP [6][7]. By making utilize of DT-PDP, the taking after three security prerequisites can be fulfilled: (1) Encourage security of the unregistered information of the obtained company can be ensured; (2) The judgment and secrecy of recorded information can be ensured; (3) The information transportability calculation can be exchanged to an outside cloud server. For the DT-PDP security concept, we grant its legitimization, its framework show, and its security model [9]. Following this, we plan a particular DT-PDP circuit based on bilinear sets. At last, we analyze the security, adequacy, and adaptability of a specific DT-PDP conspire. Safely scrambled data with lawful deduplication in cloud reencryption is utilized to keep absent from privateness data spillage and moreover to keep absence from the deduplication in a consistent position re-encryption framework (SRRS) [8]. Additionally checks for confirmation of possession to recognize whether the client is an authorized client or not. The part re-encryption strategy is to share the get to key for the comparing authorized client for getting to the specific record without the spillage of private information. To our extent, we're the utilization of each the shirking of literary substance and virtual pictures. For case, we have individual pictures on our versatile, handheld gadgets, and on the desktop, etc., so, as these images have to be kept secure and so we are utilizing encryption to extend the tall security. Intrusionresilient open cloud inspecting plot with authenticator supplant Key-publicity strong cloud carport examining can make steady cloud carport prior than and after the key-publicity term, Be that as it may, the pernicious cloud server can, in any case, alter with or indeed dispose of the client's archives which may be transferred all through the key-publicity term without being detected[10]. To address this, we offer an intrusion-proof open cloud observing conspire where the observing authenticators are upgraded frequently to prevent a malevolent cloud from disturbing these records employing a key.

Safe Cloud Storage with Reliable and Reliable Key Disclosure Control When auditing cloud storage, main disclosure is a major security risk [11]. A cloud capacity examining plot with key-exposure solidness has been proposed as an arrangement for this issue. Be that as it may, in the event that the noxious cloud gets the current private key from the information proprietor, it can parody true blue confirmation tokens after the key divulgence period [12]. In this paper, we propose a novel model for safe cloud storage testing called the powerful and robust key discovery test, in which the security of cloud capacity testing can be accomplished not as it were some time recently but moreover after revelation. We'll type in an essential construction and codify the depiction and security demonstration of this modern sort of cloud capacity testing. The security of cloud capacity reviewing at other times is unaffected by the central arrangement of getting to in our proposed conspire. The burden of information administration for information proprietors can be significantly decreased by attribute-based cloud information judgment examining for secure outsourced capacity such as cloud capacity. In spite of the numerous benefits of cloud capacity, it moreover postures a few security dangers [13]. The basic pillar of outsourcing services is data confidentiality, which is one of the foremost troublesome issues of steady cloud storage. External information checking logs empower the analyst to proficiently check the keenness of submitted records without having to download the whole record from the cloud, bringing down the contact overhead between the cloud server and the verifier we're trying to find. Execute attribute-based cloud reviewing to overcome the overpowering issue of cloud wellbeing review key management. Users can transfer records to the cloud with a custom set of qualities and 
select a set of keen analysts for outside information approval [14]. This new building block's framework and security model defines a protocol for verifying the integrity of cloud data based on unique attributes [15].

\section{Proposed System}

Within the proposed framework, a cloud capacity inspecting conspire has been executed. The proposed conspire employments the thought of combining direct blunder redress codes and direct homomorphic confirmation plans. This integration employments only one extra square to realize mistake resilience and verification at the same time. To illustrate the capabilities of the common plan, we too give a nitty-gritty plot based on the proposed common plan utilizing the Reed-Solomon code and the MAC verification plot based on widespread hash and Galois effective computation field based on GF (28). We moreover appear that the proposed conspire is secure by the standard definition. In expansion, we have actualized the proposed framework and made it accessible as an open-source arrangement. The test comes about to appear that the proposed circuit is a few orders of greatness more effective than the circuit of the earlier craftsmanship (Figure 1). In proposed system crowd source has been implemented using we propose a novel dispersed agent-based privacy-preserving system, called DADP, that presents a modern level of different operators between the clients and the untrusted server. Rather than specifically uploading the check-in data to the untrusted server, a client can arbitrarily select one operator and transfer the check-in data to it with the mysterious association innovation.

\subsection{Advantages}

-User doesn't need to know the private key.

-Better guard security.

-These deduplication systems can support differential authorization duplication checks.

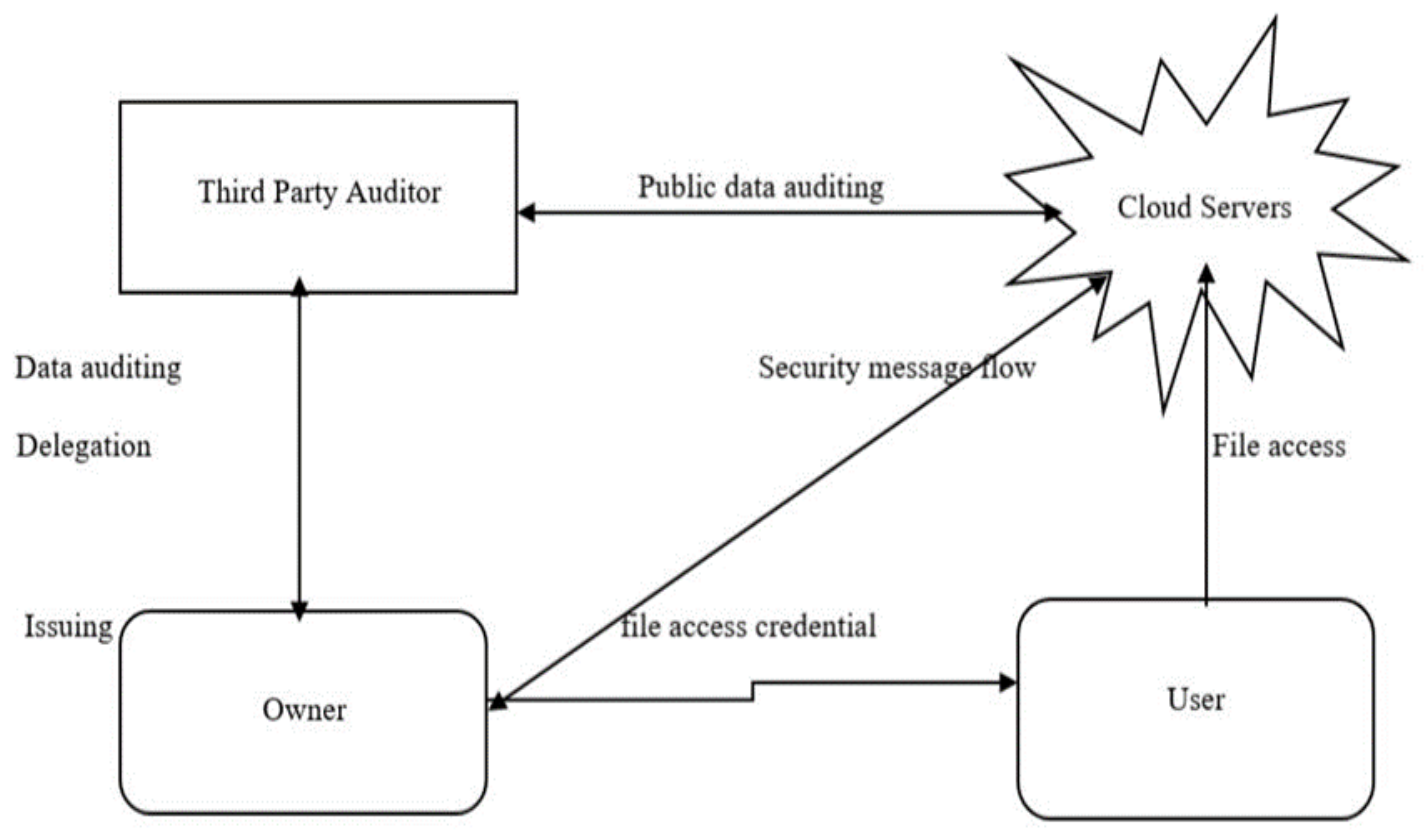

Figure 1: Proposed system

\section{System Model}

The framework demonstrate comprises of three sorts of substances: cloud, client, and office server (AS). (1) Cloud: The cloud has tremendous capacity space and gives capacity and download administrations to clients. (2) Client: The client is separated into two categories. One is the beginning client who transfers records that did not exist within the cloud already. The other one is the consequent clients who transfer records that the cloud has kept. The introductory client creates the authenticators for each encrypted record, and after that transfers the scrambled record, its comparing authenticators, and the record tag to the cloud. The taking after the client does not get to make information authenticators and does not ought to transfer these messages to the cloud. After that, the primary client and the other client can get their information after downloading the information from the cloud. Too, clients can confirm the astuteness of cloud information by running the cloud capacity observing convention with the cloud. To make strides in capacity proficiency, the cloud performs deduplication for copy records. In other words, the cloud keeps as it were a single duplicate of any copied record, and it is comparing authenticators and gives clients an interface to the comparing. (3) AS: The AS is dependable for making a difference clients produce the record file and the le name with his private key. With the file, the cloud can confirm whether the record transferred by the client is copied or not. Utilizing the record tag, the client can create keys for encryption and produce an authenticator. 


\section{UML Diagram}

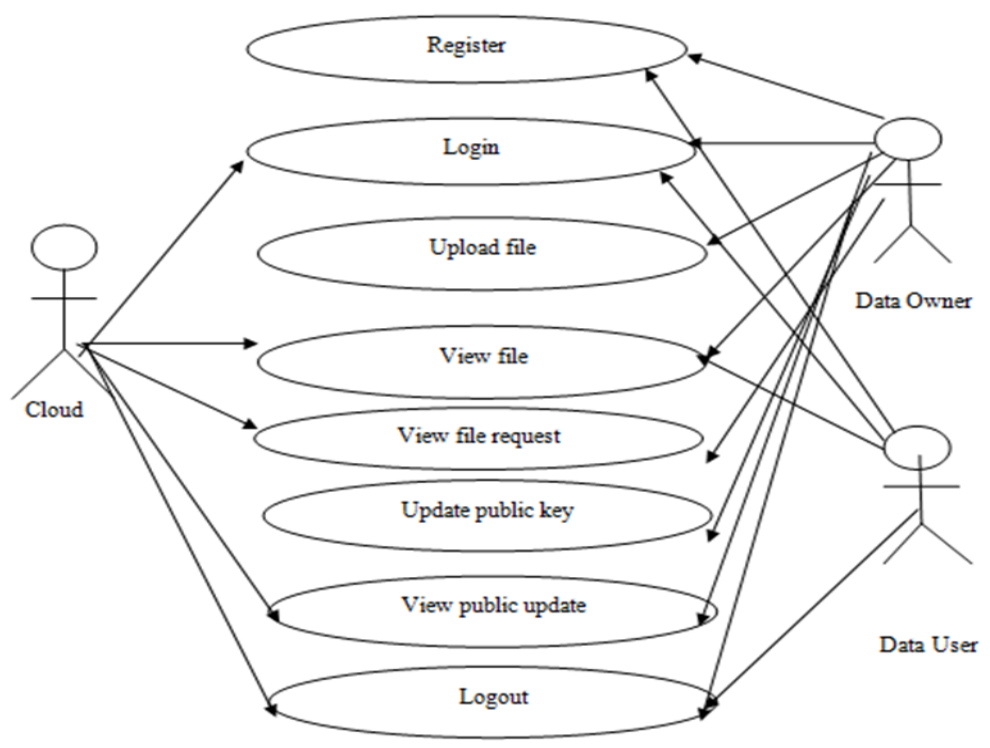

Figure 2: Use Case Diagram

This figure represents the Use case diagram of this paper and it is showing the login and registration process of the web site that we have been created (Figure 2).

\section{Class Diagram}

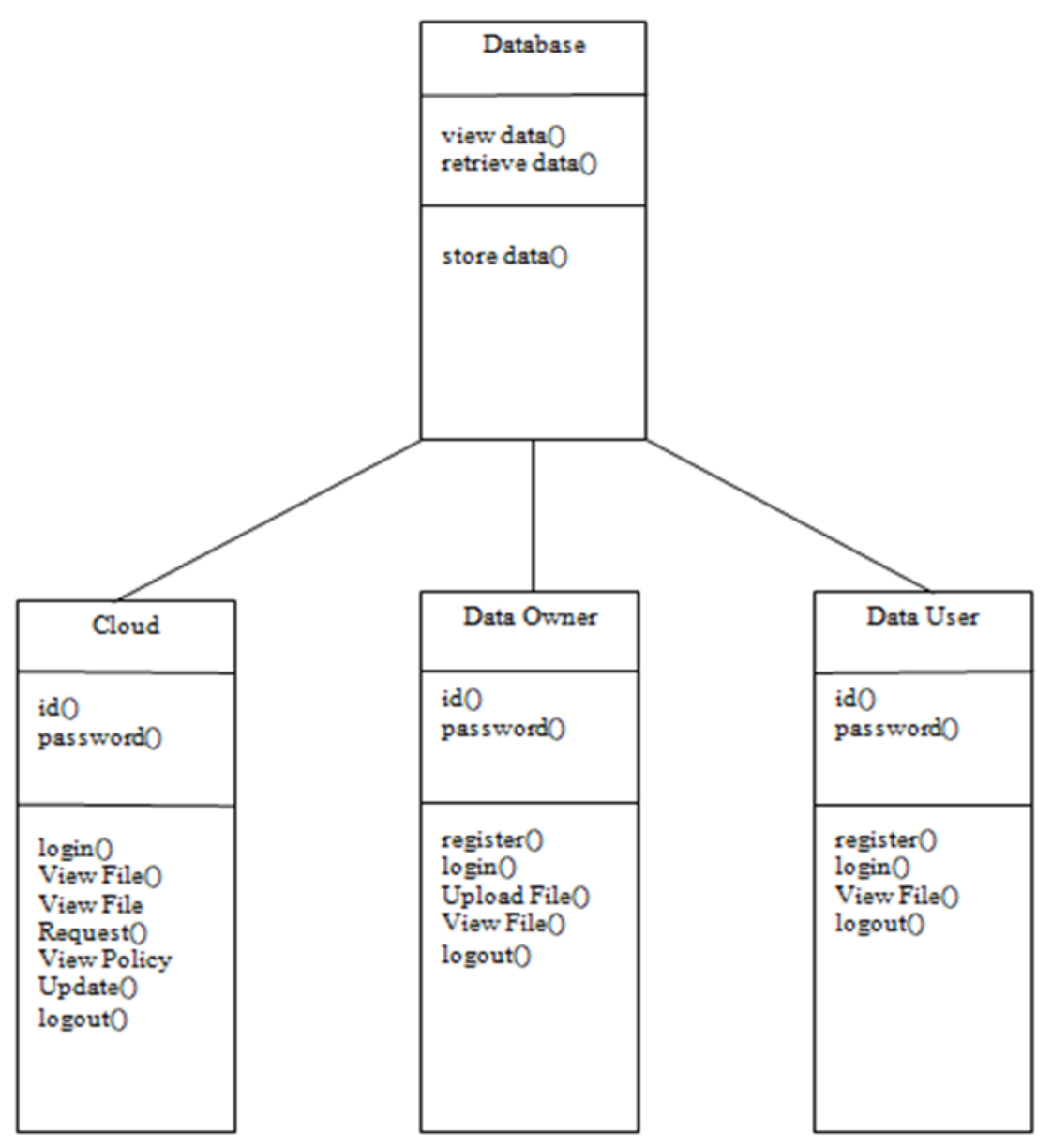

Figure 3: Class Diagram

This figure represents the Class diagram of this paper and it is showing the phases for the website to be logged in, uploading file, view file and then finally logged out (Figure 3). 


\section{Application}

\section{CtrlS Real Cloud:}

The control model of the CtrlS Real Cloud is multi-layered. Anything from application layout to root access to a digital device can be managed through a user interface and API with a cloud controller server. With Real Cloud, you may host and control programs remotely with maximum ease.

Cloud Layer Services:

Find the guarantee of the cloud, not the compromises. Cloud Layer incorporates virtual servers, farther capacity, and a vigorous substance conveyance arrange that leverages our key assets and longstanding authority in robotized, self-managing, and on-demand foundation.

\section{Result and discussion}

We proposed a dispersed agent-based privacy-preserving system, called DADP, for real-time crowd-sourced information distributing with the untrusted server. A modern level of numerous operators was presented between clients and the untrusted server to total and irritate information with differential protection in a dispersed way. We proposed a few instruments in DADP to realize suitable budget allotment for gathering and annoyance on each specialist. We demonstrated that DADP fulfills w-event -differential protection with such a conveyed system beneath the untrusted server.

\section{Conclusion}

In this paper, we raise the ease of use issue of existing cloud capacity examining plans. To illuminate this issue, we proposed a modern common cloud capacity inspecting plot based on existing cloud capacity examining plans that utilize PoR and PDP. The proposed plot coordinating mistake rectification and homomorphic confirmation in as it were one extra square; it too jams the organize of the outsourced information. The proposed conspire endures little information debasements and bolsters existing cloud capacity applications. We too instantiated the common development utilizing the Reed Solomon code and the UMAC homomorphic confirmation conspire over GF(28). The test assessment affirmed that the proposed conspire is more productive than existing plans that utilize huge numbers operations. We trust these three properties make the proposed cloud capacity reviewing conspire more usable. The test comes about on two real-world datasets that appear that DADP accomplishes nearly the same utility as Protected, and outflanks BA and BD essentially. All of these compared calculations give w-event- differential security with the worldwide data beneath the trusted server, whereas DADP is the primary work, to the finest our information, that realizes w-event -differential security for real-time crowd-sourced measurement calculation and distributing beneath the untrusted server. In specific, the utility of DADP is vigorous to the alter of the security budget, the window measure (w), and the number of operators.

\section{References}

1. S. Deoras, "8 cloud outages that shook the tech world in 2019 ," https://analyticsindiamag.com/8cloudoutages-that shook- the-tech-world-in-2019/, 2019.

2. H. Sarmah, "Cloud outages that shook the tech world: 2018," https://analyticsindiamag.com/cloudoutages-that-shook-the-tech-world-2018/, 2018.

3. H. Tian, Y. Chen, H. Jiang, Y. Huang, F. Nan, and Y. Chen, "Public auditing for trusted cloud storage services,” IEEE Security \& Privacy, vol. 17, no. 1, pp. 10-22, Jan 2019.

4. M. Sookhak, F. R. Yu, and A. Y. Zomaya, "Auditing big data storage in cloud computing using divide and conquer tables," IEEE Transactions on Parallel and Distributed Systems, vol. 29, no. 5, pp. 9991012, May 2018.

5. H. Wang, D. He, J. Yu, and Z. Wang, "Unconditional incentive based on identity and anonymous ownership of verifiable public data, "IEEE Transactions on Services Computing, vol. 12, no. 5, pp. 824$835,2019$.

6. Y. Luo, M. Xu, K. Huang, D. Wang, and S. Fu, "Efficient review of shared data in the cloud with secure user recall and IT outsourcing," Computers \& Security, vol. 73, pp. 492- 506, 2018.

7. L. Zhou, A. Fu, S. Yu, M. Su, and B. Kuang, "Verification of the integrity of big data transferred to the cloud: a study, a journal of network applications and computers, vol. 122, pp. 1 - 15, 2018.

8. Santhi, P., Priyanka, T.,Smart India agricultural information reterival system, International Journal of Advanced Science and Technology, 2020, 29(7 Special Issue), pp. 1169-1175.

9. W. Shen, J. Qin, J. Yu, R. Hao, and J. Hu, "Enable identity-based integrity checking and data exchange with hidden confidential information for secure cloud storage," IEEE Transactions on Information Forensics and Security, vol. 14, no. 2, pp. 331-346, Feb 2019.

A. Juels and B. S. Kaliski Jr, "Pors: Proofs of retrievability for large files," in Prof. of ACM CCS. Acm, 2007, pp. 584597. 
10. Santhi, P., Mahalakshmi, G., Classification of magnetic resonance images using eight directions gray level co-occurrence matrix $(8 \mathrm{dglcm})$ based feature extraction, International Journal of Engineering and Advanced Technology, 2019, 8(4), pp. 839-846.

11. M. A. Shah, R. Swaminathan, M. Baker, et al., "Controlling data protection and digital content extraction. "Archive of ePrint IACR encryption, vol. 2008, p. 186, 2008.

12. Q. Wang, C. Wang, K. Ren, W. Lou, and J. Li, "Provision of publicly available audits and data dynamics for cloud storage security, "IEEE transactions in parallel and distributed systems". , vol. 22, no. 5, pp. 847-859, 2010.

13. C. Wang, K. Ren, W. Lou, and J. Li, “Towards public and secure cloud storage services, ” IEEE Network, vol. 24, no. 4, pp. 19-24, 2010.

14. K.Deepa, S.Thilagamani, "Segmentation Techniques for Overlapped Latent Fingerprint Matching", International Journal of Innovative Technology and Exploring Engineering (IJITEE), ISSN: 2278-3075, Volume-8 Issue-12, October 2019. DOI: 10.35940/ijitee.L2863.1081219

15. Z. Hao, S. Zhong, and N. Yu, "A remote data integrity monitoring protocol that protects confidentiality through data dynamics and public auditing capabilities," IEEE Transactions on Knowledge and Data Engineering, vol. 23, no. 9, pp. 1432-1437, 2011.

16. Y. Zhu, G.-J. Ahn, H. Hu, S. S. Yau, H. G. An, and C.-J. Hu, "Dynamic audit services for outsourced storages in clouds," IEEE Transactions on Services Computing, vol. 6, no. 2, pp. 227-238, 2011.

17. Y. Zhu, H. Hu, G.-J. Ahn, and M. Yu, "Shared Verifiable Data Ownership for Integrity Verification in Multi-Cloud Storage," IEEE Transactions in parallel and distributed systems, vol. 23, no. 12, pp. 22312244, 2012.

18. Deepa, K., Kokila, M., Nandhini, A., Pavethra, A., Umadevi, M. "Rainfall prediction using CNN", International Journal of Advanced Science and Technology, 2020, 29(7 Special Issue), pp. 1623-1627. http://sersc.org/journals/index.php/IJAST/article/view/10849

19. H. Shacham and B. Waters, "Compact recoverability test," Journal of Cryptology, vol. 26, no. 3, pp. 442483, 2013.

20. Murugesan, M., Thilagamani, S. ," Efficient anomaly detection in surveillance videos based on multi layer perception recurrent neural network", Journal of Microprocessors and Microsystems, Volume 79, Issue November 2020, https://doi.org/10.1016/j.micpro.2020.103303

21. C. C. Erway, A. K"upc, " u, C. Papamanthou, and R. Tamassia, "Dynamic provable data possession," ACM Transactions on Information and System Security, vol. 17, no. 4, p. 15, 2015.

22. Thilagamani, S., Nandhakumar, C. ." Implementing green revolution for organic plant forming using KNN-classification technique", International Journal of Advanced Science and Technology, Volume 29 , Isuue 7S, pp. 1707-1712

23. D. He, S. Zeadally, and L. Wu, "Certificateless public auditingscheme for cloud-assisted wireless body area networks," IEEESystem Journal, vol. 12, no. 1, pp. 64-73, 2018.

24. S.Nakamoto,"Bitcoin: A peer-to-peer electronic cash system."https://bitcoin.org/bitcoin.pdf.

25. G. Wood, "Ethereum: A secure decentralized generalized transactionledger,"Ethereum Project Yellow Paper, vol. 151, 2014.

26. Thilagamani, S., Shanti, N.," Gaussian and gabor filter approach for object segmentation", Journal of Computing and Information Science in Engineering, 2014, 14(2), 021006, https://doi.org/10.1115/1.4026458

27. S. S. Al-Riyami and K. G. Paterson, "Certificateless public-key cryptography," in Proc. of ASIACRYPT, 2003, pp. 452-473.

28. Rhagini, A., Thilagamani, S. ,'Women defence system for detecting interpersonal crimes",International Journal of Advanced Science and Technology, 2020, Volume 29,Issue7S, pp. 1669-1675

29. Juels and B. S. K. Jr, "Pors: Proofs of retrievability for largefiles," in Proc. of ACM CCS, 2007, pp. 583-597.

30. P. Pandiaraja, N Deepa 2019," A Novel Data Privacy-Preserving Protocol for Multi-data Users by using genetic algorithm", Journal of Soft Computing, Springer, Volume 23 ,Issue 18, Pages 8539-8553,

31. S. Worku, C. Xu, J. Zhao, and X. He, "Secure and efficient privacy-preserving public auditing scheme for cloud storage," Computers\& Electrical Engineering, vol. 40, no. 5, pp. 1703-1713, 2014.

32. N Deepa , P. Pandiaraja, 2020 ," Hybrid Context Aware Recommendation System for E-Health Care by merkle hash tree from cloud using evolutionary algorithm", Journal of Soft Computing, Springer , Volume 24 ,Issue 10, Pages 7149-7161. 
33. L. Zhang, B. Qin, Q. Wu, and F. Zhang, "Efficient many-to-oneauthentication with certificate fewer aggregate signatures," ComputerNetwork, vol. 54, pp. 2482-2491, 2010.

34. N Deepa , P. Pandiaraja, 2020 , "E health care data privacy preserving efficient file retrieval from the cloud service provider using attribute based file encryption ", Journal of Ambient Intelligence and Humanized Computing, Springer, https://doi.org/10.1007/s12652-020-01911-5.

35. Y. Zhang, X. Lin, and C. Xu, "Blockchain-based secure data sources for cloud storage," in Proc. ICICS, 2018, pp. 3-19.

36. K Sumathi, P Pandiaraja 2019," Dynamic alternate buffer switching and congestion control in wireless multimedia sensor networks", Journal of Peer-to-Peer Networking and Applications, Springer, Volume 13,Issue 6,Pages 2001-2010..

37. R. C. Merkle, "Protocols for public-key cryptosystems," in Proc. ofIEEE S \& P, 1980, pp. 122-134.

38. J. Garay, A. Kiayias, and N. Leonardos, "The bitcoin backboneprotocol:Analysis and applications," in Proc. EUROCRYPT, 2015,pp. 281-310.

39. Vijayakumar, P, Pandiaraja, P, Balamurugan, B \& Karuppiah, M 2019, 'A Novel Performance enhancing Task Scheduling Algorithm for Cloud based E-Health Environment', International Journal of E-Health and Medical Communications (IJEHMC), Vol 10,Issue 2,pp 102-117..

40. Y. Zhang and M. Blanton, "Efficient dynamic provable possession of remote data via update trees," ACM Trans. Storage, vol. 12, no. 2, 2016, Art. no. 9.

41. Vijayakumar, P ,Pandiaraja, P, , Karuppiah, M \& Deborah, LJ 2017, 'An Efficient Secure Communication for Healthcare System using Wearable Devices', Journal of Computers and Electrical Engineering, Elsevier, Vol .No 63, October 2017, pp 232-245.

42. H. Zhao, X. Yao, X. Zheng, T. Qiu, and H. Ning, "User stateless privacy-preserving TPA auditing scheme for cloud storage," J. Netw. Comput. Appl., vol. 129, pp. 62-70, 2019.

43. B. Chen and R. Curtmola, "Remote data integrity checking with server-side repair 1," J. Comput. Secure., vol. 25, no. 6, pp. 537-584, 2017.

44. Y. Zhang, C. Xu, X. Lin, and X. S. Shen, "Blockchain-based Public Integrity Checker for cloud storage of slow listeners," IEEE Trans. Cloud Comput., to be published, DOI: 10.1109/TCC.2019.2908400.

45. H. Wang, H. Qin, M. Zhao, X. Wei, H. Shen, and W. Susilo, "Blockchain-based fair payment smart contract for public cloud storage auditing," Inf. Sci., vol. 519, pp. 348-362, 2020.

46. Fu, Y. Li, S. Yu, Y. Yu, and G. Zhang, "DIPOR: An idea-based dynamic proof of retrievability scheme for cloud storage systems," J. Netw. Comput. Appl., vol. 104, pp. 97-106, 2018.

47. Wikipedia, "Systematic code," 2019. [Online]. Available: https://en.wikipedia.org/wiki/Systematic_code

48. M. N.Wegman and J. L. Carter, "New hash functions and their use in authentication and set equality," J. Comput. Syst. Sci., vol. 22, no. 3, pp. 265-279, 1981.

49. F. Meng, "Source code for experimental evaluation," 2019. [Online]. Available: https://github.com/fchen-group/auditing-with-error-correction 